\title{
Early visual deprivation does not prevent the emergence of basic numerical abilities in blind children.
}

\begin{abstract}
Studies involving congenitally blind adults demonstrated that visual experience is not a mandatory prerequisite for the emergence of efficient numerical abilities. It remains however unknown whether blind adults developed lifelong strategies to compensate for the absence of foundations vision would provide in infancy. We therefore assessed basic numerical abilities in blind and sighted children of 6 to 13 years old. We also assessed verbal and spatial working memory abilities and their relationship with mental arithmetic in both groups. Blind children showed similar or better numerical abilities as compared to the sighted. Blind children also outperformed their sighted peers in every task assessing verbal working memory and demonstrated a similar spatial span. The correlation between arithmetic and the spatial sketchpad was affected by the group while the correlations between arithmetic and the other two components (the central executive and the phonological loop) were not affected by early visual experience. Our data suggest that early blindness does not impair the development of basic numerical competencies in children but influences the associations between arithmetic and some working memory components.
\end{abstract}

Keywords: numbers, blind children, arithmetic, estimation, counting, memory. 


\section{Introduction}

It has been suggested that the foundations of numerical processing were rooted in general visuospatial mechanisms (Burr \& Ross, 2008; Ross \& Burr, 2010; Simon, 1999; Stoianov \& Zorzi, 2012). If the representation of numerical magnitudes actually relies on a visuo-spatial medium, how would numerical abilities develop in people born blind? Some recent studies suggested that visual deprivation could affect number representation and its processing (see Crollen \& Collignon, 2020 for a review). Blind individuals were for example shown to use the finger-counting strategy less spontaneously than their sighted peers (Crollen, Mahe, Collignon, \& Seron, 2011; Crollen, Noël, Seron, Mahau, Lepore, \& Collignon, 2014). Blindness was similarly shown to affect the nature of the spatial reference frame in which the spatial processing of numbers occurs (Crollen, Dormal, Seron, Lepore, \& Collignon, 2013). Early visual deprivation was finally shown to shape the neural circuitry of mathematical reasoning (Amalric, Denghien, \& Dehaene, 2018; Crollen, Lazzouni, Bellemare, Rezk, Lepore, Noël, Seron, \& Collignon, 2019; Kanjlia, Lane, Feigenson, \& Bedny, 2016). Indeed, several studies found similar brain activation in the classic number-responsive prefrontal and intraparietal regions while blind and sighted people perform arithmetic operations (Amalric, Denghien, \& Dehaene, 2018; Crollen, Lazzouni, Rezk, Bellemare, Lepore, Noël, Seron, \& Collignon, 2019; Kanjlia, Lane, Feigenson, \& Bedny, 2016). However, and in contrast to sighted individuals, congenitally blind people additionally activate their occipital cortex during numerical thinking. While the additional recruitment of this visual region by the blind has been used as an evidence that the occipital cortex is functionally flexible early in life (Lane, Kanjlia, Omaki, \& Bedny, 2015) and capable of repurposing itself toward unrelated cognitive functions (Kanjlia et al., 2016; Bedny, 2017), another study recently assumed that this activation may actually depend on the intrinsic computational role of the activated regions (Crollen et al., 2019).

Despite these qualitative differences between blind and sighted adults in the way they process numbers, the lack of vision did not preclude the development of various numerical skills (Castronovo, 2014). Blind and sighted people were for example shown to present the same bisection effects in numerical bisection tasks (Cattaneo, Fantino, Silvanto, Tinti, \& Vecchi, 2011; Rinaldi, Vecchi, Fantino, Merabet, \& Cattaneo, 2015). They appeared to behave similarly in tasks involving the haptic exploration 
of a rod in order to indicate its midpoint, while listening to a particular number (Cattaneo, Fantino, Tinti, Silvanto, \& Vecchi, 2010). They showed the same subitizing range as their sighted peers (Ferrand, Riggs, \& Castronovo, 2010) and, perhaps more surprisingly, even demonstrated better numerosity estimation (Castronovo \& Delvenne, 2013; Castronovo \& Seron, 2007; Ferrand et al., 2010; Togoli, Crollen, Arrighi, \& Collignon, 2020), counting (Crollen et al., 2014) and calculation skills (Dormal et al., 2016) as compared to the sighted. Altogether, these results therefore support the idea that numerical skills can emerge despite a lack of visual experience.

The majority of existing data on numerical performances following early blindness however comes from adults. It therefore remains unclear whether basic numerical abilities are equally good in sighted and blind children or if, in contrast, blind children underperform their sighted peers but reach the same level of performance as the typical population in adulthood only. Indeed, it might be that blind children would suffer from the lack of foundations vision typically provides for the development of numeracy. It is indeed possible that blind children need to progressively develop alternative learning mechanisms and strategies to compensate the absence of vision so that, in adulthood, they reach a performance level comparable to the one of the sighted. With this unresolved question in mind, we therefore evaluated the performance of blind and sighted children in a series of basic visuo-spatial numerical tasks, already tested in adults (number-to-position, number bisection, counting, and mental arithmetic). If blindness induces a delay in numerical development, then blind children should show lower performances than their sighted peers in basic numerical skills. In contrast, if early visual experience is not mandatory to develop good numerical skills, then blind children should show similar (or even better) numerical performances than sighted children.

In sighted, a large number of studies have moreover pointed out the role of working memory (WM) in numerical cognition and more particularly in arithmetic development (Andersson \& Lyxell, 2007; Geary, 2005; Noël, 2009; Noël, Seron, \& Trovarelli, 2004; Rasmussen \& Bisanz, 2005). Most of these studies were based on the architecture of Baddeley and Hitch (1974) WM model. According to this model, WM is mainly divided into three subsystems: the central executive component (CE) that allows accessing information retained in the WM; the phonological loop, related to the verbal working 
memory (e.g. the retention of verbal information); the visuo-spatial working memory, designed to retain and process visuo-spatial information. While a reliable association has been observed between the central executive and arithmetic problem solving (Bull \& Scerif, 2001; De Smedt, Janssen, Bouwens, Verschaffel, Boets, \& Ghesquiere, 2009; Gathercole \& Pickering, 2000; Geary, Hamson, \& Hoard, 2000; Noël, 2009; Passolunghi, Vercelloni, \& Schadee, 2007), the association between arithmetic and the 2 slave systems remains debated in the literature. While some associations have been found between the visuo-spatial sketchpad and numerical abilities (Holmes, Adams, \& Hamilton, 2008; Jarvis \& Gathercole, 2003), the role of the phonological loop in solving arithmetic operations is less clear. Most studies with Western participants have indeed failed to observe a significant implication of this component (e.g., Bull \& Johnston, 1997; De Smedt et al., 2009; De Rammelaere, Stuyven, \& Vandierendonck, 2001; De Rammelaere, \& Vandierendonck, 2001; DeStefano \& LeFevre, 2004; Gathercole \& Pickering, 2000; Geary et al., 2000; Holmes \& Adams, 2006; Noël et al., 2004; Seitz \& Schumann-Hengsteler, 2000). Some other studies suggest that the central executive and the phonological loop facilitate performance during early stages of mathematical learning whereas visuospatial representations play an increasingly important role during later stages (Meyer, Salimpoor, $\mathrm{Wu}$, Geary, \& Menon, 2010).

The second aim of the present paper was therefore to study the associations between arithmetic and the 3 WM components in blind children. Indeed, it has been assumed that blind people may compensate for their lack of vision in the development of their arithmetic skills by relying on enhanced WM abilities (Castronovo \& Delvenne, 2013; Crollen et al., 2011; Crollen, Collignon, \& Noël, 2017; Kanjlia, Feigenson, \& Bedny, 2018; Salillas, Granà, El-Yagoubi, \& Semenza, 2009) but the three WM components and their links with arithmetic development have never been systematically and directly tested in blind children. We therefore asked our participants to perform several WM tasks and evaluated the relationships between this cognitive function and arithmetic processing. Are the associations between WM and arithmetic the same in blind and sighted children or are they affected by early visual deprivation? 


\section{Methods and results}

\section{Participants}

Twelve congenitally blind (CB) children ( 7 girls and 5 boys; 8 right-handed, 3 ambidextrous) participated in the study. All were born with visual impairment and did not have any other disability. Six children were recruited in the French speaking part of Belgium, 5 were recruited in France and 1 in Switzerland. The children were between 6 and 12 years old and presented different causes of blindness (see Table 1 for a detailed description of the children). Nine blind children out of the 12 tested were integrated in typical schools while the others were in special schools. Sixteen sighted children (SC) between 6 and 13 years old (11 girls, 5 boys; all right-handed) were recruited in Belgium and in France from the same typical classrooms as the blind participants. IQ of both groups was estimated by two verbal subtests of the Wechsler Intelligence Scale for Children-IV (Wechsler, 2003): Similarity and Information. Because these subtests were representative of the verbal scale, the mean of their raw scores was calculated and compared across groups. Sighted children were matched to the blind in terms of IQ $[M \pm S E=19.71 \pm 1.30$ for the blind; $M \pm S E=16.00 \pm 1.57$ for the sighted; $t(26)=-1.73, p>.09]$ and age $[M \pm S E=9.58 \pm 0.62$ years for the blind; $M \pm S E=9 \pm 0.58$ for the sighted; $t(26)=-0.68, p>.5]$. All test procedures were approved by the research ethics board of the Catholic University of Louvain (UCLouvain, Belgium). Written informed parental consent was obtained for all of the children. Children were tested twice, each testing session lasting approximately one hour.

Table 1. Characteristics of the blind children.

\begin{tabular}{|c|c|c|c|c|c|c|}
\hline Participants & Gender & Age & Handedness & Onset & Cause of blindness & $\begin{array}{c}\text { Visual } \\
\text { perception }\end{array}$ \\
\hline $\mathrm{CB} 1$ & $\mathrm{~F}$ & 11 & $\mathrm{R}$ & 0 & Aplasia of the optic nerves & No \\
\hline $\mathrm{CB} 2$ & M & 11 & $\mathrm{R}$ & 0 & Aplasia of the optic nerves & No \\
\hline CB3 & $\mathrm{F}$ & 8 & $\mathrm{R}$ & 0 & Premature child & Lights \\
\hline CB4 & $\mathrm{F}$ & 9 & $\mathrm{R}$ & 0 & Malformation at birth & $\begin{array}{c}\text { Mass } \\
\text { perception }\end{array}$ \\
\hline
\end{tabular}




\begin{tabular}{ccccccc}
\hline CB5 & F & 6 & R & 3 months & Aplasia of the optic nerves & Lights and \\
shapes
\end{tabular}

\section{Procedure and data analysis}

Four memory tasks (pseudo-words repetition, Corsi block tapping, listening span, catego span) and 4 numerical tasks (number bisection, number-to-position, counting, arithmetic) were presented to the children. The order of presentation of the tasks was counterbalanced across participants. The sighted children perform every task with a blindfold, therefore in conditions similar to those of the blind participants. Moreover, as the spatial sketchpad and the number-to-position tasks involved a modality (touch) that is generally less used by the sighted to solve such tasks, the same sighted participants additionally performed this task with the eyes opened

The methods and data analysis of each task will be described in details below. Results of the different tasks were analyzed with parametric tests. This allowed us to include, in the same statistical test, the between-subject factor of group and the age as a covariate. However, as some of our data were not normally distributed, non-parametric tests (Mann-Withney U) were also conducted in order to further assess the presence of groups' differences. These non-parametric analyses are reported in the 
supplementary Table 1 and lead to the exact same conclusions as the parametric tests reported in the main text of this manuscript.

\section{Numerical tasks}

\section{Number bisection.}

In this task, 24 pairs of numbers were auditorily presented to the children. Children received the instruction to judge and orally report the numerical midpoint of the number pair as quickly as possible without calculating. Each number pair was presented twice: once in ascending order (i.e., smallest number presented first and largest number presented afterwards) and once in descending order (i.e., smallest number presented after the largest number). Two levels of difficulty were created. Children from 6 to 9 years old were required to perform a simple version of the task. In this version, the number pairs included a one-digit number and a two-digit number. The numerical distance between both numbers was either 7, 9 or 11 . In the difficult version of the task, half of the pairs included a one-digit number and a two-digit number; the other half included 2 two-digit numbers. The numerical distance between the numbers was either 11, 13 or 15. E-Prime (Psychology Software Tools, Pittsburgh, PA, USA) was used to present the stimuli. Reaction times were collected and the experimenter recorded children's responses. The Percentage of Error (PE) for each number pair was calculated as follows: ((participant's number midline estimation - true midline)/size of the interval)*100. Negative values indicated a left bias, and positive values indicated a right bias. The $|\mathrm{PAE}|$ (i.e., absolute value of the PE) was also computed. One sighted child as well as one blind child were not included in the analyses as they were unable to perform the task.

The PE were first submitted to a one-sample t-test (reference value $=0$ ) to examine whether children's responses were under- or overestimated. Overall, there was a pseudo-neglect effect $(M \pm S E$ $=-9.25 \pm 3.95), t(25)=-2.34, p=.02$. When compared to each other, the two groups did not differ $(M$ $\pm S E=-3.95 \pm 3.78$ for the blind; $M \pm S E=-13,15 \pm 6.19$ for the sighted), $t(24)=-1.16, p=.26$.

An ANCOVA, with presentation order (ascending vs. descending) and distances (level 1, level 2, level 3) as within-subject factors, the group (blind vs. sighted) as the between-participant factor and 
the age as a covariate was then carried out on the PAE. Results only showed a significant distance $\mathrm{x}$ group interaction, $F(2,46)=4.76, p=.03, \eta^{2}{ }^{2}=.17$, suggesting that blind performed better $(M \pm S E=$ $18.66 \pm 5.91)$ than sighted $(M \pm S E=29.28 \pm 9.16)$ in the first distance level only, $t(24)=2.35, p=.03$

(see Figure 2A, left panel). There was no group difference in the other 2 distance levels $\left(p_{s}>.3\right)$ The age and group effects were not significant, $F(1,23)=0.62, p=.44, \eta_{P}^{2}=.03$ for the age effect; $F(1,23)$ $=2.84, p=.10, \eta^{2}=.11$ for the group effect.

The same ANCOVA performed on the reaction times however revealed a significant effect of group, $F(1,23)=10.54, p=.004, \eta_{P}^{2}=.31:$ blind children were faster $(M \pm S E=3484.87 \mathrm{~ms} \pm 964.13)$ than the sighted $(M \pm S E=7607.93 \mathrm{~ms} \pm 825.54)$. No other significant results were found (see Figure $2 \mathrm{~A}$, right panel).

\section{Number-to-position}

A tactile number-to-position task was created with a $25 \mathrm{~cm}$ graduated rule positioned on a wooden board (see Figure 1B). Children were told that they needed to show where they thought different numbers would fall on the rule by moving a cursor to the estimated location. According to the age of the children, the rule was verbally labeled " 0 " at its left end and either " 20 " (for 6 to 9 -year old children), "100" (for 10- and 11-year old children) or "1000" (for 12 and 13-year old children) at its right end (see supplemental table 1 for a list of the auditory stimuli presented in each condition of the task). There were no time restrictions. Responses were recorded by the experimenter who was the only one able to see the graduations of the rule. The deviations to the true number's position were carefully measured. The Percentage of Error (PE) for each number was computed as follows: ((participant's number estimation - true number)/line's scale)*100. Negative values indicated a left bias, and positive values indicated a right bias. The Percentage of Absolute Error (i.e., PAE) was computed as well by computing the absolute value of the PE. Thirtheen sighted children (out of the 16) also performed the task without the blindfold. The three other children were not tested with the eyes opened due to time constraints. 


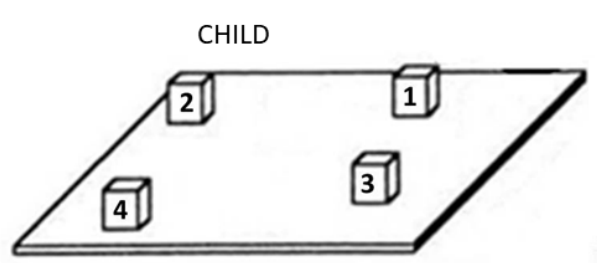

EXPERIMENTER

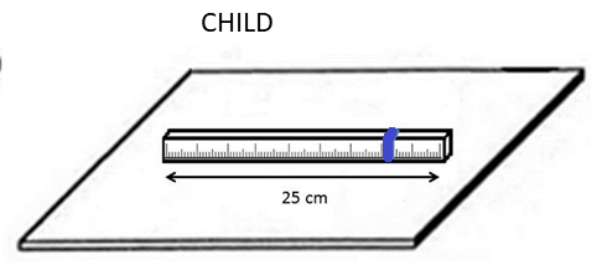

EXPERIMENTER

Figure 1. (A) Tactile adaptation of the Corsi block tapping task. (B) Tactile number-to-position task.

To examine children's left or right bias, the Percentage of Error (PE) was first submitted to a one-sample $\mathrm{t}$ test with 0 as the reference value. The mean PE was $-4.44(\mathrm{SE}=1.83)$, which was statistically different from $0, t(27)=-2.42, p=.02$. Children therefore underestimated the true location of the numbers on the rule. An independent samples t-test moreover demonstrated that the underestimation was similar in both sighted and blind $(M \pm S E=-2.54 \pm 2.45$ for the blind; $M \pm S E=$ $5.87 \pm 2.65$ for the sighted), $t(26)=-0.89, p=.38$.

Then, to analyze the precision of children's responses, an ANCOVA with group (SC with the blindfold vs. CB) as the between-subject factor and age as a covariate was conducted on children's PAE. This analysis did not demonstrate any significant effect, $F(1,25)=1.07, p=.31, \eta^{2}{ }_{P}=.04$ for the age factor; $F(1,25)=1.49, p=.23, \eta^{2} P=.06$ for the group variable.

In the sighted group, children obtained larger PAE in the tactile condition of the task $(M \pm S E=$ $14.52 \pm 1.86)$ as compared to the visual one $(M \pm S E=12.03 \pm 2.30), t(12)=2.44, p=.03$. Tactile PAE of the blind $(M \pm S E=11.49 \pm 1.38)$ was finally similar to the visual PAE of the sighted $(M \pm S E=$ $12.03 \pm 2.30), t(23)=0.20, p=.85($ see Figure $2 \mathrm{~B})$

\section{Counting.}

To assess counting abilities, we used exactly the same procedure as in Crollen et al. (2011). Children were exposed to 10 series of two phonetically dissimilar syllables (/pa/ and /ji/) and were instructed to count the numbers of /pa/ and /ji/ sounds in a sequence (e.g., "“/pa/, /ji/, /ji/, /pa/, /pa/, /ji/"'= $3 / \mathrm{pa} /, 3 / \mathrm{ji} /$ ). The syllables were emitted at a rate of one per second. The total number of syllables ranged from 6 to 10; the number of /pa/ sounds ranged from 3 to 7, and the number of /ji/ sounds ranged from 2 to 6 . One point was given when the child was able to give the correct number of $/ \mathrm{pa} / \mathrm{and}$ the number 
of /ji/ within a sequence. The number of children using the finger-counting strategy was also assessed in each group. One blind child was removed from the analyses because he didn't want to try the task.

An ANCOVA with group as the between-subject factor and age as a covariate was first conducted on the number of finger-counting uses and revealed a significant effect of group, $F(1,24)=$ $15.40, p=.001, \eta^{2}=.39$, but no significant effect of age, $F(1,24)=0.004, p>.9, \eta^{2}{ }_{P}=.000$. While all sighted children used their fingers, only five blind children did it (one child out of the 5 used this procedure only once). Two of the blind children tested were twins, but only one of these children used the finger-counting strategy while the other one didn't.

The same ANCOVA, performed this time on the accuracy scores, only showed a marginal effect of age, $F(1,24)=3.03, p=.09, \eta_{P}^{2}=.12$, and a marginal effect of group, $F(1,24)=3.03, p=.09, \eta_{P}^{2}$ $=.11$. Counting was therefore similarly performed by sighted $(M \pm S E=7.94 \pm 0.64)$ and blind children $(M \pm S E=6.55 \pm 0.77)($ see figure $2 \mathrm{C})$.

\section{Mental arithmetic.}

Children were asked to perform 10 additions, 10 subtractions and 20 multiplication problems (see Table 2 for a detailed list of the operations used). All problems were presented auditorily at a comfort intensity level. To record reaction times (RTs), the experimenter pressed a space bar as soon as the child responded. Children were required to answer as fast and as accurately as possible by giving an oral answer. Responses were encoded by the experimenter who also controlled the presentation of the next trial by pressing the space bar (to make sure that the child was focused on the task). Two levels of difficulty were used for the addition and subtraction operations. Children from 6 to 9 years old were required to perform 8 "one-digit + one-digit" operations and 2 "one-digit + two-digit" additions including one with carryover. Children from 10 to 13 years old were required to perform more complicated operations: 4 "two-digit + one-digit" additions including 2 with carryover and 6 "two-digit + two-digit" operations including 4 with carryover. Subtraction problems were created directly on the basis of the addition problems by taking the sum as the minuend and one of the addend as the subtrahend (see Table 2). All the children performed the multiplication task but were stopped after 5 incorrect 
answers. The younger children ( $\mathrm{N}=2$ in the sighted group and $\mathrm{N}=1$ in the blind group) were unable to perform the multiplication task. As the youngest children ( 1 blind and 2 sighted) did not perform the multiplication task, we separately analyzed the data of addition and subtraction on the one hand and multiplication on the other hand.

\section{Addition and subtraction}

A 2 (operation: addition, subtraction) x 2 (group: SC vs. CB) ANCOVA was first performed on the percentage of correct responses (i.e., ((number of correct responses/total number of items)*100) with age as a covariate. This analysis highlighted a main effect of group, $F(1,25)=15.42, p=.001, \eta^{2} P^{=}$ .38. Blind children $(M \pm S E=87.96 \pm 5.01)$ outperformed their sighted peers $(M \pm S E=61.84 \pm 4.33)$. The effect of age was marginally significant, $F(1,25)=3.08, p=.09, \eta^{2}{ }_{P}=.11$

The same ANCOVA performed this time on the reaction times (expressed in ms) yielded the same results by showing a significant group effect, $F(1,25)=8.81, p=.007, \eta^{2}{ }_{P}=.26$. Reaction times for addition and subtraction were faster in the blind $(M \pm S E=5021.13 \mathrm{~ms} \pm 1648.87)$ than in the sighted $(M \pm S E=11520.56 \mathrm{~ms} \pm 1426.19)($ see Figure 2D). The age effect was marginally significant, $F(1,25)$ $=3.75, p=.06, \eta^{2}{ }^{2}=.13$. No other effect or interactions were significant.

\section{Multiplication}

An ANCOVA with age as covariate was conducted to analyse children's percentage of correct responses in the multiplication task. The only significant effect was the main effect of age, $F(1,25)=$ 12.06, $p=.002, \eta_{P}^{2}=.35$. The group effect was not significant, $F(1,25)=0.59, p=.45, \eta_{P}^{2}=.03$. However, when the analysis was performed on the reaction times, we observed a main effect of age $\left(F(1,25)=9.43, p=.006, \eta_{P}^{2}=.30\right)$ showing that $\mathrm{RT}$ in the multiplication task decreases with age. A main effect of group was moreover observed, $F(1,25)=17.62, p=.0001, \eta_{P}^{2}=.44$, showing that blind children $(M \pm S E=3692.19 \mathrm{~ms} \pm 1010.10)$ responded faster than their sighted peers $(M \pm S E=9385.92$ $\mathrm{ms} \pm 894.18$ ) (see Figure 2D). 
Table 2. List of the operations presented to the children.

\begin{tabular}{ccc|c}
\hline Difficulty level & Additions & Subtractions & Multiplications \\
\hline & $2+7$ & $9-7$ & $2 * 4$ \\
& $4+1$ & $5-1$ & $4 * 8$ \\
& $2+4$ & $6-4$ & $7 * 2$ \\
Simple operations & $3+5$ & $8-5$ & $3 * 9$ \\
From 6 to 9 years old & $5+4$ & $9-4$ & $6 * 9$ \\
& $8+2$ & $10-2$ & $3 * 6$ \\
& $7+6$ & $13-6$ & $8 * 5$ \\
& $9+3$ & $12-3$ & $4 * 3$ \\
& $12+4$ & $16-4$ & $9 * 8$ \\
& $14+7$ & $21-7$ & $5 * 7$ \\
& $12+5$ & $17-5$ & $12 * 4$ \\
Difficult operations & $42+6$ & $48-6$ & $21 * 3$ \\
& $34+9$ & $43-9$ & $13 * 7$ \\
& $56+7$ & $63-7$ & $43 * 2$ \\
& $21+48$ & $69-48$ & $11 * 5$ \\
& $65+34$ & $99-34$ & $16 * 6$ \\
& $17+16$ & $33-16$ & $27 * 3$ \\
& $38+47$ & $85-47$ & $38 * 2$ \\
& $49+75$ & $124-75$ & $18 * 4$ \\
& $53+89$ & $142-89$ & $12 * 8$ \\
\hline
\end{tabular}


A. Number bisection PAE

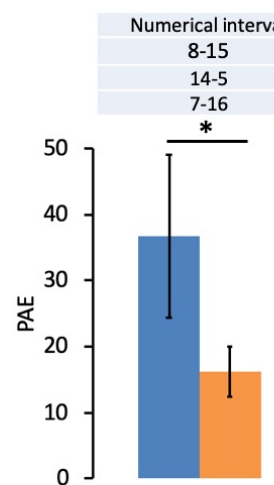

D1

C. Number-to-position PAE
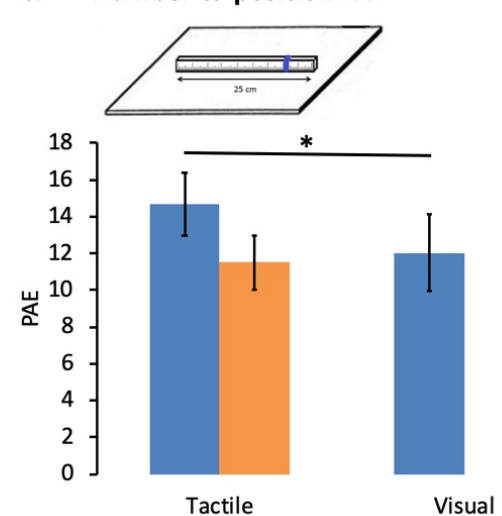

E. Arithmetic ACC

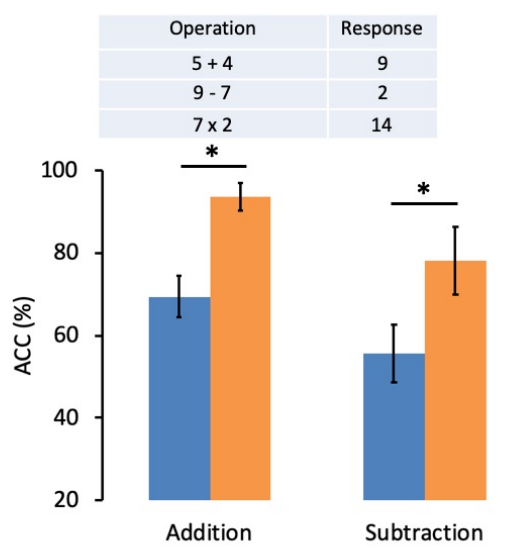

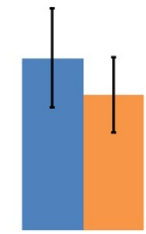

D3

B. Number bisection RT

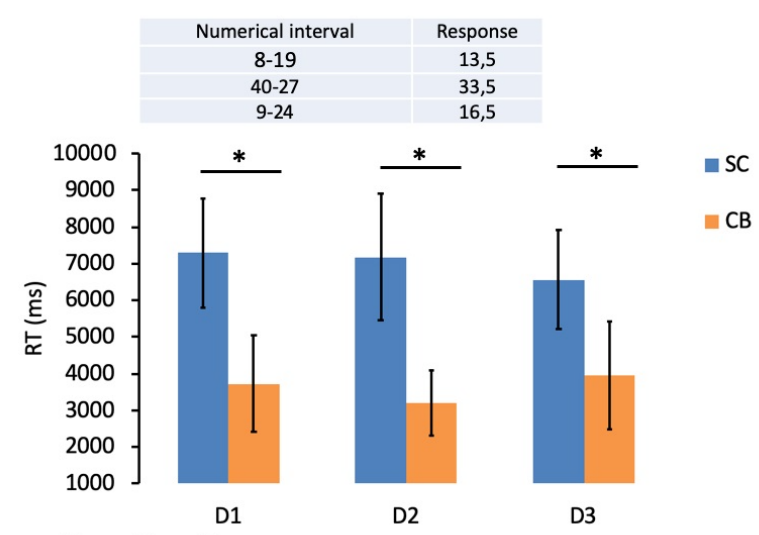

D. Counting
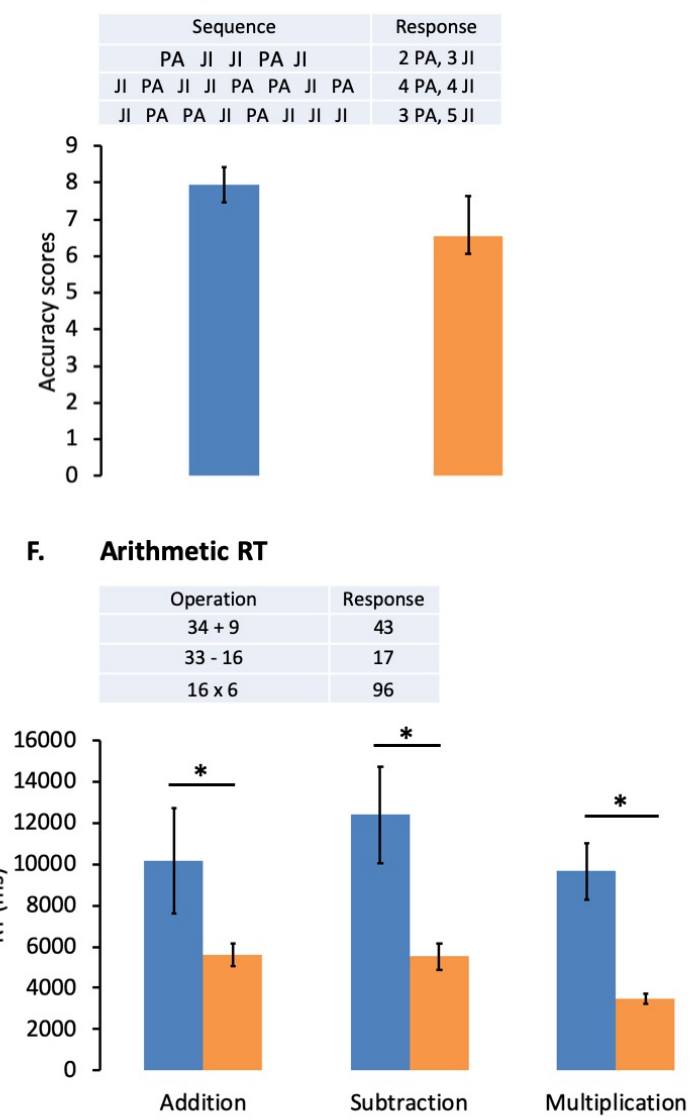

Figure 2. Numerical performances of the sighted ( $\mathrm{SC}$, in blue) and congenitally blind ( $\mathrm{CB}$, in orange) children. (A) Percentage of absolute error - PAE - in the number bisection task; (B) Reaction Times RT - in the number bisection task. D1, D2 and D3 represent the sizes of the numerical intervals presented. (C) PAE in the number-to-position task. Sighted children performed the task with a blindfold (tactile condition) and with the eyes opened (visual condition); (D) Accuracy scores in the counting task (maximum score $=10)$; (E) Accuracy (ACC - in percentages) in the different operations (addition, subtraction, multiplication) of the arithmetic task; (F) Reaction times - RT - in the different operations (addition, subtraction, multiplication) of the arithmetic task. Examples of stimuli or material are 
represented below the title of each task. Bars represent standard error of the mean. Significant results are highlighted by an asterisk $(*)$.

\section{Memory tasks}

Children's working memory capacities were examined using tasks evaluating each specific components of the working memory system: the phonological loop, the spatial sketchpad, and the central executive.

\section{Phonological loop.}

The capacity to store verbal material was assessed through a task that did not involve number words and the child's mental lexicon: the pseudo-word repetition task. It is part of the standardized French BELEC battery (Mousty, Leybaert, Alegria, Content, \& Morais, 1994). The test included two pseudo-word lists differing in terms of syllabic complexity level (consonant-vowel [CV] for the first list and consonant-consonant-vowel $[\mathrm{CCV}]$ for the second list). The task started with a two-syllable non-word to repeat (four trials) and was followed by longer non-words of three, four, and five syllables. One point was given for each correctly repeated non-words, giving a total maximum score of 20 for each list. The task was stopped after 4 consecutive errors.

A 2 (condition: $\mathrm{CV}$ vs. $\mathrm{CCV}$ ) x 2 (groups: SC vs. CB) ANCOVA with age as a covariate was run on the pseudo-words repetition scores. This analysis showed an effect of condition, $F(1,25)=24.04$, $p<.001, \eta_{p}^{2}=0.49$, a significant effect of group, $F(1,25)=4.51, p<.05, \eta_{p}^{2}=0.15$, and a significant effect of age, $F(1,25)=5.24, p<.05, \eta_{p}^{2}=0.17$. Performances in the CV list $(M \pm S E=18.16 \pm 0.33)$ were better than in the CCV one $(M \pm S E=11.45 \pm 0.48)$. Blind children $(M \pm S E=15.58 \pm 0.55)$ performed better than their sighted peers $(M \pm S E=14.03 \pm 0.48)$ and total score improved with age. No other effect or interaction were significant (see Figure 3A).

\section{(Tactile)-Spatial sketchpad.}

The Corsi block-tapping task is a widely used test to assess visuo-spatial working memory. Here we adapted this test in the tactile modality. The test was administered using four square blocks 
positioned on a wooden board (see Figure 3A). First, children were required to touch the blocks in order to create a representation of their location on the board. Then, the experimenter took the dominant hand of the children and tapped the blocks according to a specific order. When the tapping sequence was terminated, children's hand was repositioned on a specific position on the table and participants were required to perform the same sequence of movements, in the same order as the one done with the experimenter. The sequence started out simple but became progressively more complex (from 2 to 7 movements). There were two trials at each level of difficulty. If the child failed these two trials, the task was stopped. Each corrected sequence was credited with 0.5 point. Sighted children first performed the task with a blindfold and then with the eyes opened.

An ANCOVA with group (SC in the blindfold condition vs. $\mathrm{CB}$ ) as the between-subject factor and age as a covariate was first performed to compare groups' performance in the tactile modality. This analysis did not yield any significant results, demonstrating that both groups behaved similarly $(M \pm S E$ $=3.24 \pm 0.34$ for the blind; $M \pm S E=2.78 \pm 0.29$ for the sighted), $F(1,25)=1.17, p>.2, \eta_{p}^{2}=.04$, and that spatial memory did not improve with age, $F(1,25)=1.39, p>.2, \eta^{2}{ }_{p}=.05$. The same analysis was then performed to compare the blind performance in the tactile modality to the sighted performance in the visual modality. The group difference was not significant, $F(1,25)=0.54, p>.4, \eta^{2} p=0.02$. Again, there was no effect of age, $F(1,25)=2.24, p>.1, \eta_{p}^{2}=0.08$. A paired-samples t-test finally highlighted that sighted children reached a higher level of accuracy when doing the task with the eyes open $(M \pm$ $S E=3.57 \pm 0.29)$ as compared to blindfolded, $t(15)=-3.57, p=.003$ (see Figure 3B).

\section{Central executive.}

Two complex span tasks were used to assess children's central executive capacities (the listening span test and the catego-span task). In the listening span task (Censabella \& Noël, 2008), the experimenter read a set of sentences to the children, who were instructed first to judge the truth value of the sentences and then to recall, in any order, the last word of all the sentences in the set. Half of the sentences were true (e.g., "Le boulanger fait du pain"' [The baker makes bread]); the other half were false (e.g., "Les tabourets ont des dents" [Stools have teeth]). The number of sentences read to the 
children progressively increased (from 2 to six). There were three trials at each level of difficulty. If the child failed at two out of the three trials, the task was stopped. The corrected span was used as the dependent measure. This is the longest sequence for which two series were repeated correctly, plus 0.5 point if one longer series was also correctly processed.

In the catego-span task (Noël, 2009), one-syllable food or animal words were presented to the child, who was asked to repeat them by category, giving the foods first and then the animals or the animals first and then the foods. The administration and correction procedures were the same as the ones used in the listening span task - that is, three trials per length and calculation of the corrected span.

An ANCOVA with group ( $\mathrm{SC}$ vs. $\mathrm{CB}$ ) as the between-subject factor and age as a covariate was first performed on the listening span measure. Results showed a significant effect of group, $F(1,25)=$ $10.75, p=.003, \eta_{p}^{2}=0.30$, meaning that blind children $(M \pm S E=3.73 \pm 0.29)$ obtained higher scores than their sighted peers $(M \pm S E=2.48 \pm 0.25)$ (see Figure 3C) and a significant effect of age, $F(1,25)$ $=4.22, p=.05, \eta_{p}^{2}=0.14$, suggesting that the listening span measure tended to increase with age. The same ANCOVA was then conducted on the category-span measure and showed a significant effect of group, $F(1,25)=8.55, p=.007, \eta_{p}^{2}=0.25$, again indicating better performance in the blind children $(M \pm S E=5.25 \pm 0.30)$ than in the sighted ones $(M \pm S E=4.19 \pm 0.20)($ See Figure 3D). The age effect was not significant, $F(1,25)=1.07, p>.3, \eta_{p}^{2}=0.04$ 
A. Pseudo-words repetition task

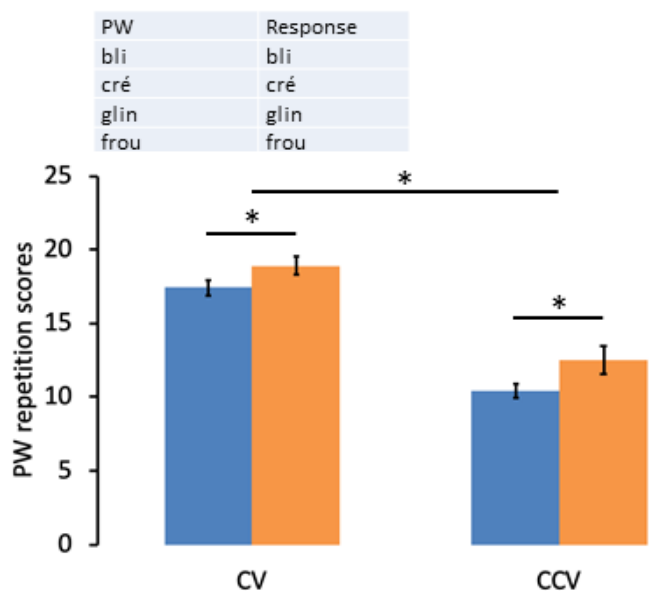

C. Listening span
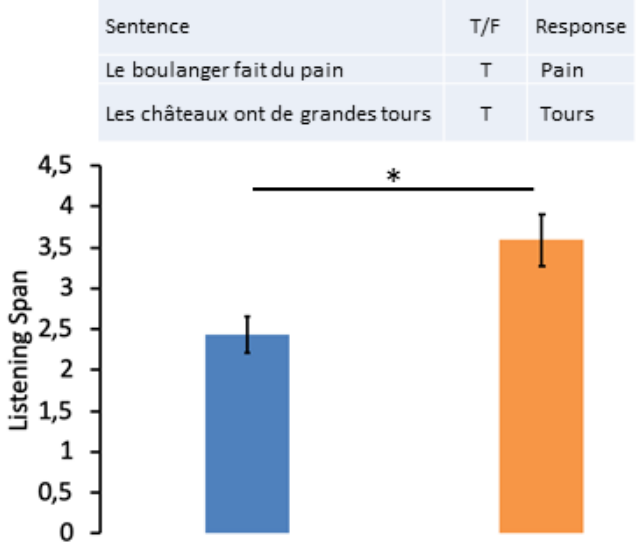

B. Tactile spatial sketchpad

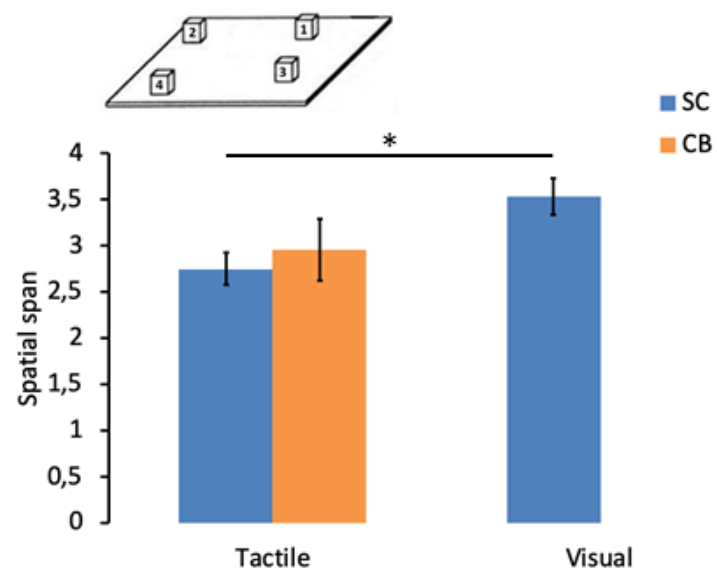

D. Catego span
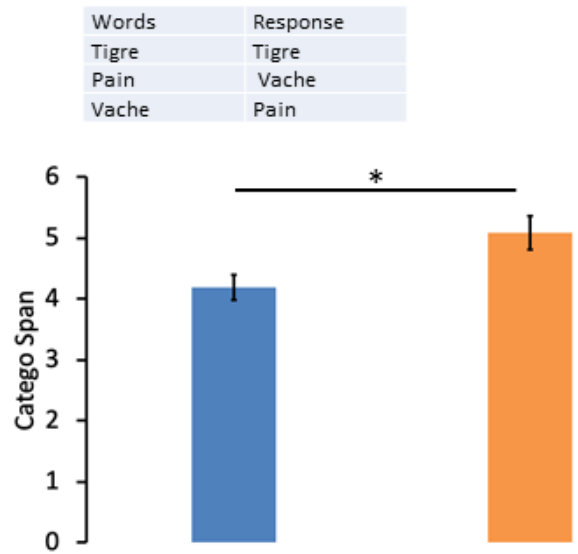

Figure 3. Memory performances of the sighted ( $\mathrm{SC}$, in blue) and blind ( $\mathrm{CB}$, in orange) children. (A) Verbal span in the pseudo-words repetition task; (B) Spatial span in the spatial sketchpad task. Sighted children performed the task with the blindfold (tactile condition) and with the eyes opened (visual condition); (C) Listening span score; (D) Catego span measure. Examples of stimuli or material are represented below the title of each experiment. Bars represent standard error of the mean. Significant results are highlighted by an asterisk $(*)$.

\section{Correlations between mental arithmetic and working memory}

Correlations analyses (unilateral Spearman r) were then computed in each group separately to examine whether memory and arithmetic scores (percentage of correct responses) were associated with each other.

To calculate the correlations with a reduced number of analyses, we computed a global arithmetic score by computing first the z-scores of each participant in each arithmetical task (addition, 
subtraction, multiplication) and then computing the mean of these $\mathrm{z}$ scores for each individual ${ }^{1}$. Similarly, a central executive composite score was calculated by calculating for each participant, the mean of his/her z-scores in the listening and catego span tasks. As 3 correlations were calculated in each group, the corrected $\mathrm{p}$ level is .017 .

In the sighted group, the central executive measure tended to be correlated with the arithmetic performances. However, the p-value was just above the significance level of .017 (i.e., .019, see Table 3). In the blind group, arithmetic correlated with the central executive as well as with the spatial span. No correlation emerged with the pseudo-word repetition task, in any of the 2 groups (see Table 3). To further examine whether the correlation between WM and arithmetic differs in the 2 groups, moderator analyses were computed. As shown in Tables 4-6, the effect of the moderator (group) was significant for the 3 WM components but the interaction between arithmetic and group was only significant for the spatial span measure. This result suggests that the correlation between arithmetic and the spatial sketchpad was affected by the group while the correlations between arithmetic and the other 2 components (the central executive and the phonological loop) were not affected by the presence or absence of early visual experience. This analysis included all the participants, even those who did not complete the multiplication operation (i.e., 2 sighted and 1 blind). The composite score of these children only included their addition and subtraction performances. However, the exact same conclusions were reached when the correlations were performed without these children.

Table 3. Correlations between arithmetic performances and memory measures in the sighted and blind groups.

\begin{tabular}{lccc}
\hline & Central executive & Corsi & PW \\
\hline Arithmetic in the SIGHTED & $.52(p=.019)$ & $-.18(p>.2)$ & $.26(\mathrm{p}>.1)$ \\
Arithmetic in the BLIND & $.84(p<.001)$ & $.64(p=.013)$ & $.40(\mathrm{p}=.09)$ \\
\hline
\end{tabular}

$\mathrm{PW}=$ Pseudo-Words repetition task

\footnotetext{
${ }^{1}$ The formula for calculating a $\mathrm{z}$-score is $\mathrm{z}=(\mathrm{x}-\mu) / \sigma$, where $\mathrm{x}$ is the raw score, $\mu$ is the population mean, and $\sigma$ is the population standard deviation
} 
Table 4. Relationship between arithmetic and the central executive using group as the moderator

\section{Arithmetic}

$\begin{array}{lccccc}\text { Constant } & 33.83 & 7.62 & 4.44 & \mathbf{. 0 0 0} & \\ \text { Group } & 23.07 & 5.01 & 4.60 & \mathbf{. 0 0 0} & 0.63 \\ \text { Central executive } & 10.67 & 2.69 & 3.97 & \mathbf{. 0 0 1} & \\ \text { Central executive x Group } & 4.47 & 2.77 & 1.61 & .122 & \end{array}$

Table 5. Relationship between arithmetic and the spatial span using group as the moderator

\section{Arithmetic}

$\begin{array}{lccccc}\text { Constant } & 34.93 & 9.11 & 3.83 & \mathbf{. 0 0 1} & \\ \text { Group } & 22.06 & 5.98 & 3.69 & \mathbf{. 0 0 1} & 0.47 \\ \text { Spatial span } & 2.71 & 2.83 & 0.96 & .350 & \\ \text { Spatial Span x Group } & 6.37 & 2.88 & 2.21 & \mathbf{. 0 4} & \end{array}$

Table 6. Relationship between arithmetic and the phonological loop using group as the moderator

\begin{tabular}{lccccc}
\hline Arithmetic & $\boldsymbol{b}$ & $\boldsymbol{S E} \boldsymbol{B}$ & $\boldsymbol{t}$ & $\boldsymbol{p}$ & $\boldsymbol{R}^{\mathbf{2}}$ \\
Constant & & & & & \\
Group & 33.05 & 10.29 & 3.21 & $\mathbf{. 0 0 4}$ & \\
Phonological loop & 23.78 & 6.71 & 3.54 & $\mathbf{. 0 0 2}$ & 0.39 \\
Phonological loop x Group & 4.72 & 4.92 & 0.96 & .348 & \\
& 3.73 & 4.78 & 0.78 & .444 &
\end{tabular}
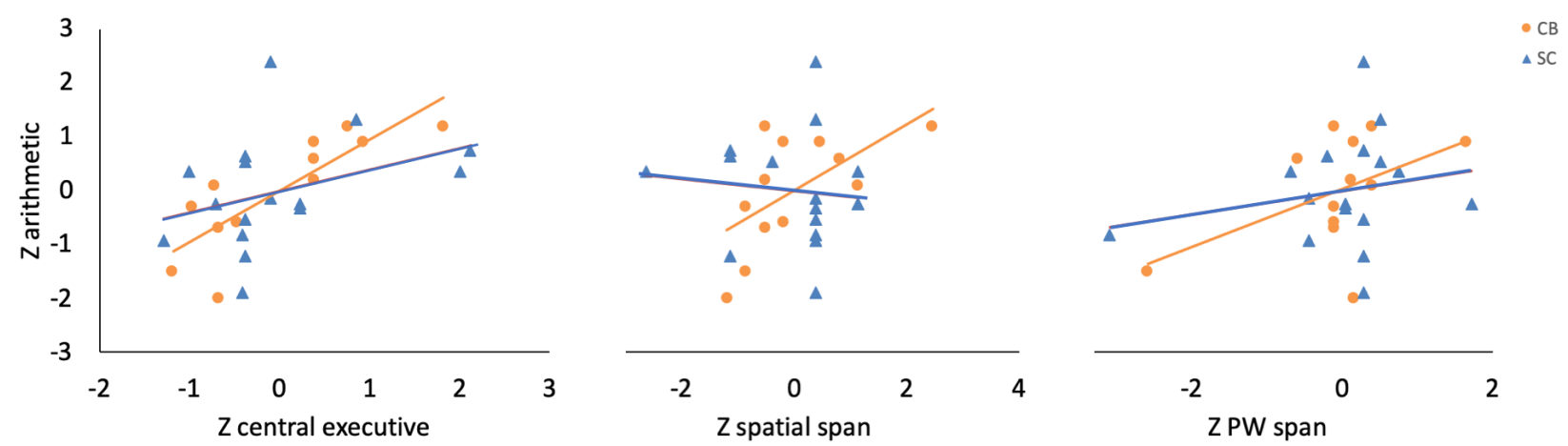

Figure 4. Spearman correlations between the Z-scores of the arithmetic and memory tasks (central executive on the left, spatial sketchpad in the middle and pseudo-word repetition on the right) of blind (in orange) and sighted children (in blue). 


\section{Discussion}

Vision has for a long time been suggested to play a central role in the development of numerical cognition. In contrast to other sensory modalities, vision indeed allows the precise and simultaneous processing of a large amount of information. As sequential processing was found to be more complex than simultaneous numerical processing in children (Mix, 1999), adults (Tokita \& Ishiguchi, 2012) and animals (Nieder, Diester, \& Tudusciuc, 2006), vision has been suggested to provide the foundational attribute of number representation and skills (Burr \& Ross, 2008; Stoianov \& Zorzi, 2012). But, is vision necessary to develop optimal numerical abilities? Over the past decade, a growing body of evidence has challenged this idea by demonstrating that early blindness was not an obstacle for the observation of good numerical abilities in blind individuals. However, if numerical abilities of blind adults were shown to be comparable or even sometimes superior to the one of the sighted, little was known about the emergence of these abilities in blind children. It might indeed be that blind children need to progressively develop compensatory learning mechanisms and strategies to deal with the absence of vision. In our study, we compared basic numerical abilities of blind and sighted children. Moreover, as working memory has been linked to the development of good arithmetic skills in sighted children (Andersson \& Lyxell, 2007; Geary, 2005; Noël, 2009; Noël et al., 2004; Rasmussen \& Bisanz, 2005), we also assessed working memory skills and their relationship with mental arithmetic in sighted as well as in blind children.

We observed that blind children presented similar or even better performances than their sighted peers in all the numerical tasks tested, and independently of their age, the cause of blindness and the remaining visual perception (see supplemental figure 1). In the number bisection, number-to-position, and counting tasks, blind children indeed behaved similarly than the sighted children. Interestingly, in the number-to-position task, the blind's scores in the tactile modality were equivalent to the ones of the sighted performing the task in the visual modality. Blind and sighted children, like adults (Blini, Cattaneo \& Vallar, 2013; Cattaneo et al., 2010, 2011; Rinaldi et al., 2015), were moreover shown to present the same pseudo-neglect effect in the number bisection and number-to-position tasks. This last observation suggests that visual experience is not mandatory for the development of some characteristics 
of the mental number line (e.g., a leftward visuo-spatial bias). In the number bisection task, blind children again presented similar accuracy scores (and even better performances in the distance level 1) as compared to the sighted but, more importantly, performed the task on average 2 times faster, demonstrating that their access to number magnitude is more direct or automatized. The data of the counting task support the idea that visual experience plays an important role in implementing the sensori-motor habits that drive the development of finger-number interactions (Crollen et al., 2011, 2014). Blind children indeed used the finger-counting strategy less often than their sighted peers in order to alleviate their working memory load. Despite this strategy difference, blind and sighted children achieved a quite similar counting performance, suggesting that the use of fingers is not mandatory to achieve optimal performance in counting. Blind children finally showed better arithmetic performances (more correct responses coupled with smaller reaction times) than sighted children (see Dormal et al., 2016 for similar results in adults) in all the operations tested (addition, subtraction, multiplication). Our results therefore suggest that vision is not mandatory for the emergence of good arithmetic and basic numerical skills in children.

Regarding the working memory data, we observed that blind children outperformed their sighted peers on all the verbal memory tasks (both on the simple and on the complex span tasks). In contrast, the groups did not differ significantly on the spatial task, similarly to what has already been observed with adults (Castronovo \& Delvenne, 2013; Dormal et al., 2016; Occelli, Lacey, Stephens, Merabet, \& Sathian, 2017). Interestingly, while the sighted showed a larger spatial span when performing the task in their dominant visual modality, there was no difference between the blind tactile span and the sighted visual one. This last observation is in line with previous studies demonstrating that the performance of blind adults in a tactile working memory task was equivalent to the one of the sighted performing the task in the visual modality (Bliss, Kujala, \& Hamalaninen, 2004; Cohen, Voss, Lepore, \& Scherzer, 2010). Finally, two memory measures (central executive and spatial-span) were found to be correlated with arithmetic performances in the blind group. In contrast to what was observed in the blind, the correlation between arithmetic and the spatial span was far from significant in the sighted group. A moderator analysis further supported the idea that the group affected the correlation between the spatial 
span and the arithmetic performance. This could be explained by the fact that there are age-related differences with regard to the contribution of the memory components to the sighted mathematics performance; the visuospatial sketchpad being a unique predictor of mathematics achievement at the beginning of the learning process only (De Smedt et al., 2009; Holmes \& Adams, 2006). Accordingly, further research with a larger cohort of participants would be necessary to examine whether the difference between sighted and blind population regarding the correlations between spatial sketchpad capacities and arithmetic performance is present in older children but not in younger ones. The small sample used here prevents us from running such an analysis.

Our study is a first step towards a better understanding of the influence of blindness on numerical abilities and their links with WM components. Other questions should nevertheless be investigated in the future. It will be interesting to examine the roles played by the different components of WM in the different arithmetic operations (is the spatial span more related to the subtraction operation and the phonological loop more related to multiplication?). It will also be interesting to further investigate other models of WM such as the Attentional-Order-Short-term memory (A-O-STM) model dissociating item and order WM. Item WM is considered as a temporary activation of the identity of the items with their phonological, lexico-semantic or visuo-spatial characteristics while order WM is considered as an independent component dedicated to process the serial order of the items presented (Majerus, 2009). So far, it is still unclear whether serial order is coded along the same principles in the verbal and the visuospatial domain. A study of Ginsburg and colleagues (Ginsburg, Archambeau, van Dijck, Chetail, $\&$ Gevers, 2017) however recently suggested that serial order was coded in a domain general fashion, but only when the to-be-remembered information was processed at the semantic level. Several studies have moreover shown that item and order components relied on distinct neural networks in healthy adults (Majerus, D’Argembeau, Perez, Belayachi, Van der Linden et al., 2010) and children (Attout, Ordonez Magro, Szmalec, \& Majerus, 2018) and might be selectively impaired in patients with brain lesions (Kalm \& Norris, 2014; Majerus, Attout, Artielle, \& Van der Kaa, 2015). Specific alterations in serial order WM abilities, as opposed to item WM abilities, have for example been observed in genetic syndromes such as 22q11.2 microdeletion syndrome or Down syndrome (Brock \& Jarrold, 2005; 
Majerus, Glaser, Van der Linden, \& Eliez, 2006; Majerus, Van der Linden, Braissand, \& Eliez, 2007) as well as in learning disorders such as dyslexia (Martinez Perez, Majerus, Mahot, \& Poncelet, 2012) or dyscalculia (Attout \& Majerus, 2015; De Visscher, Szmalec, Van Der Linden, \& Noël, 2015), and this for both verbal and visuospatial material. The tasks used in the present paper do unfortunately not allow us to correctly assess the distinction between item and order WM. On the one hand, the corsi block tapping and the pseudo-word repetition tasks dot not involve the to-be-remembered information to be processed at the semantic level (Ginsburg et al., 2017). On the other hand, the listening span and the catego span measures do not require participants to repeat the items in the correct order. Future studies should therefore be specifically designed to examine the distinction between item and order WM components. We think that it's particularly important to investigate this distinction in the verbal modality. Indeed, in the present study, there was no correlation between arithmetic and phonological loop. However, it's possible that different components of phonological loop are related to arithmetic. As order WM capacity in the verbal modality is a robust predictor of calculation abilities as compared to estimates of item WM (Attout, Noël, \& Majerus, 2014), and as blind individuals present greater order WM skills as compared to their sighted peers (Bottini, Mattioni, \& Collignon, 2016; Raz, Striem, Pundak, Orlov, \& Zohary, 2007), it would be worth examining in the future whether the blind numerical skills could be accounted by the use of enhanced verbal order WM.

As visual deprivation induces the use of auditory and tactile information, blind people constantly use serial-memory strategies in order to acquire knowledge about the world. For example, the blind navigate the world by forming sequential representations of their environment. Blind people also rely on serial-tactile strategies to identify objects. This could in turn improve their order WM processes and therefore boost their arithmetic development. As both item and serial order WM tasks have been associated with a left frontoparietal network which has been proposed to support attentional control during WM tasks (Majerus, Péters, Bouffier, Cowan, \& Phillips, 2018; Todd \& Marois, 2004) and as blind people were shown to present better selective, sustained and divided attention than their sighted peers (Collignon, Renier, Bruyer, Tranduy, \& Veraart, 2006; Pigeon et al., 2015), it would be interesting 
in the future to assess whether blind children are able to better use some specific attentional control strategies while learning arithmetic.

To sum up, our data demonstrate that blindness does not delay the development of basic numerical abilities. Blind children indeed presented similar or better numerical performances than their sighted peers. Altogether, the present data do therefore not support the "general loss hypothesis" (Gori, Sandini, Maertinoli, \& Burr, 2010; Rauschecker, 1995a,b) according to which early sensory deprivation induces a generalized degradation of sensory/cognitive functions due to the lack of calibrating role vision may provide. The present data favor the contrasting hypothesis of "compensatory adaptation". According to this hypothesis, people deprived of one sensory modality actually develop alternative compensatory sensory and cognitive abilities to process information from the remaining modalities (Collignon, Voss, Lassonde, \& Lepore, 2009; Lessard, Pare, Lepore, \& Lassonde, 1998; Voss, Lassonde, Gougoux, Fortin, Guillemot, \& Lepore, 2004). It was recently suggested that blind people may develop mathematical understanding by mostly relying on haptic manipulation (Crollen, Collignon, \& Noël, 2017) or verbal working memory processes (e.g., Castronovo \& Delvenne). Interestingly, regions of the occipital cortex that typically process visual information were shown to massively enhance their response to tactile (Reich, Szwed, Cohen, \& Amedi, 2011) and verbal information in the blind (Amedi, Raz, Pianka, Malach, \& Zohary, 2003). This reorganization of the occipital cortex (Bavelier and Neville, 2002; Sadato et al., 1996) may therefore contribute to the development of alternative strategies while blind people learn arithmetic (Crollen et al., 2019). Notwithstanding these striking findings, several key aspects of the mechanisms underlying the development of the brain plasticity remain elusive (Crollen \& Collignon, 2020) and almost nothing is known about the developmental trajectory of the recruitment of the occipital cortex when processing numbers. The study of blind infants will therefore open new windows onto our understanding of numerical development without vision. 


\section{Acknowledgments}

The authors were supported by the Belgian National Funds for Scientific Research (OC, MPN), the European Union's Horizon 2020 research and innovation program under the Marie Sklodowska-Curie grant agreement No 700057 (VC) and the 'MADVIS' European Research Council starting grant (ERCStG 337573) attributed to OC.

\section{Sample Credit author statement}

Virginie Crollen: Conceptualization, methodology, formal analysis, investigation, data curation, writing original draft, funding acquisition. Hélène Warusfel: investigation, formal analysis. MariePascale Noël: conceptualization, writing - review \& editing, supervision. Olivier Collignon: writing - review \& editing, supervision, funding acquisition.

\section{References}

Amalric, M., Denghien, I., \& Dehaene, S. (2018). On the role of visual experience in mathematical development: Evidence from blind mathematicians. Dev Cogn Neurosci. 30, 314-323. doi: 10.1016/j.den.2017.09.007.

Amedi, A., Raz, N., Pianka, P., Malach, R., \& Zohary, E. (2003). Early “visual” cortex activation correlates with superior verbal memory performance in the blind. Nat Neurosci. 6(7), 758-766.

Andersson, U., \& Lyxell, B. (2007). Working memory deficit in children with mathematical difficulties: A general or specific deficit? J Exp Child Psychol, 96(3), 197-228.

Attout, L., Majerus, S., \& Noël, M.P. (2014). The relationship between working memory for serial order and numerical development: a longitudinal study. Dev psychol, 50(6), 1667-1679.

Attout, L., \& Majerus, S. (2015). Working memory deficits in developmental dyscalculia: the importance of serial order. Child Neuropsychol, 21(4), 432-450.

Attout, L., Ordonez Magro, L., Szmalec, A., \& Majerus, S. (2019). The developmental neural subtsrates of item and serial order components of verbal working memory. Hum Brain mapp, 40(5), 1541-1553. 
Baddeley, A. D., \& Hitch, G. (1974). Working memory. In G.H. Bower (Ed.), The psychology of learning and motivation: Advances in research and theory (Vol. 8, pp. 47-89). New York: Academic Press.

Bedny, M. (2017). Evidence from blindness for a cognitively pluripotent cortex. Trends Cogn Sci, 21(9), 637-648.

Blini, E., Cattaneo, Z., \& Vallar, G. (2013). Different effects of numerical magnitude on visual and proprioceptive reference frames. Front Psychol, 4: 190.

Bliss, I., Kujala, T., \& Hämäläinen, H. (2004). Comparison of blind and sighted participants' performance in a letter recognition working memory task. Brain Res Cogn Brain Res, 18(3), 273-277.

Bottini, R., Mattioni, S., \& Collignon, O. (2016). Early blindness alters the spatial organization of verbal working memory. Cortex, 83, 271-279.

Brock, J., \& Jarrold, C. (2005). Serial order reconstruction in Down sundrome: evidence for a selective deficit in verbal short-term memory. J Child Psychol Psychiatry, 46(3), 304-316.

Bull, R., \& Johnston, R. S. (1997). Children's arithmetic difficulties: Contributions from processing speed, item identification, and short-term memory. Journal of Experimental Child Psychology, 65, 1-24.

Bull, R., \& Scerif, G. (2001).Executive functioning as a predictor of children's mathematics ability: inhibition, switching, and working memory. Dev Neuropsychol, 19(3), 273-293.

Burr, D., \& Ross, J. (2008). A visual sense of number. Curr Biol, 18, 425-428.

Castronovo, J. (2014). Numbers in the dark: Numerical cognition and blindness. In R. Cohen Kadosh, \& A. Dowker (Eds.), The Oxford Handbook of Numerical Cognition. Oxford: Oxford University Press.

Castronovo, J., \& Delevenne, J.F.,2013). Superior numerical abilities following early visual deprivation. Cortex, 49(5), 1435-1440.

Castronovo, J. \& Seron, X. (2007). Numerical estimation in blind subjects: evidence of the impact of blindness and its following experience. J Exp Psychol Hum Percept Perform, 33 (5), 10891106. 
Cattaneo, Z., Fantino, M., Silvanto, J., Tinti, C., \& Vecchi, T. (2011). Blind individuals show pseudoneglect in bisecting numerical intervals. Attent Percept Psychophys, 73, 1021-1028.

Cattaneo, Z., Fantino, M., Tinti, C., Silvanto, J., \& Vecchi, T. (2010). Crossmodal interaction between the mental number line and peripersonal haptic space representation in sighted and blind individuals. Attent Percpet Psychophys, 72 (4), 885-890.

Censabella, S., \& Noël, M.P. (2008). The inhibition capacities of children with mathematical disabilities. Child Neuropsychol, 14(1), 1-20.

Cohen, H., Voss, P., Lepore, F., \& Scherzer, P. (2010). The nature of working memory for Braille. PLoS One, 5(5), e10833.

Collignon, O., Renier, L., Bruyer, R., Tranduy, D., \& Veraart, C. (2006). Improved selective and divided spatial attention in early blind subjects. Brain Res, 1075(1), 175-182.

Collignon, O., Voss, P., Lassonde, M., \& Lepore, F. (2009). Cross-modal plasticity for the spatial processing of sounds in visually deprived subjects. Exp Brain Res, 192(3), 343-358.

Crollen, V., \& Collignon, O. (2020). How visual is the "number sense"? Insights from the blind. Neurosci Biobehav Rev, 118, 290-297.

Crollen, V., Collignon, O., \& Noël, M.P. (2017). Visuo-spatial processes as a domain-general factor of numerical development in atypical populations. Journal of Numerical Cognition, 3(2), 344364.

Crollen, V., Dormal, G., Seron, X., Lepore, F., \& Collignon, O. (2013). Embodied numbers: The role of vision in the development of number-space interactions. Cortex, 49, 276-283.

Crollen, V., Lazzouni, L., Bellemare, A., Rezk, M., Lepore, F., Noël, M.P., Seron, X., \& Collignon, O. (2019). Recruitment of the occipital cortex by arithmetic processing follows computational bias in the congenitally blind. Neuroimage, 186, 549-556.

Crollen, V., Mahe, R., Collignon, O., \& Seron, X. (2011). The role of vision in the development of finger-number interactions: Finger-counting and finger-montring in blind children. $J$ Exp Child Psychol, 109, 525-539. 
Crollen, V., Noël, M.P., Seron, X., Mahau, P., Lepore, F., \& Collignon, O. (2014). Visual experience influences the interactions between fingers and numbers. Cognition, 133, 91-96.

De Rammelaere, S., Stuyven, E., Vandierendonck, A. (2001). Verifying simple arithmetic sums and products: Are the phonological loop and the centrzal executive invovled? Mem Cognit, 29, 267273.

De Rammelaere, S., \& Vandierendonck, A. (2001). Are executive processes used to solve simple mental arithmetic production tasks? Current Psychology Letters: Behaviour, Brain, \& Cognition, 5, 7989.

De Smedt, B., Janssen, R., Bouwens, K., Verschaffel, L., Boets, B., \& Ghesquiere, P. (2009). Working memory and individual differences in mathematics achievement: A longitudinal study from first grade to second grade. J Exp Child Psychol, 103, 186-201.

DeStefano, D., \& LeFevre, J.A. (2004). The role of working memory in mental arithmetic. Europ J Cognit Psychol, 16, 353-386.

De Visscher, A., Szmalec, A., Van Der Linden, L., \& Noël, M.P. (2015). Serial order learning impairment and hypersensitivity to interference in dyscalculia. Cognition, 144, 38-48.

Dormal, V., Crollen, V., Baumans, C., Lepore, F., \& Collignon, O. (2016). Early but not late blindness leads to enhanced arithmetic and working memory abilities. Cortex, 83, 212-221.

Ferrand, L., Riggs, K., \& Castronovo, J. (2010). Subitizing in congenitally blind adults. Psychon Bull Rev, 17(6), 840-845.

Gathercole, S.E., \& Pickering, S.J. (2000). Working memory deficits in children with low achievements in the national curriculum at 7 years of age. Br J Educ Psychol, 70(2), 177-194.

Geary, D. C. (2005). Les troubles d'apprentissage en arithmétique: Rôle de la mémoire de travail et des connaissances conceptuelles. In M. P. Noël (Ed.), La dyscalculie: Troubles du développement numérique de l'enfant (pp. 169-192). Marseille, France: Solal.

Geary, D.C., Hamson, C.O., \& Hoard, M.K. (2000). Numerical and arithmetical cognition: a longitudinal study of process and concept deficits in children with learning disability. J Exp Child Psychol, 77(3), 236-263. 
Ginsburg, V., Archambeau, K., van Dijck, J.P., Chetail, F., \& Gevers, W. (2017). Coding of serial order in verbal, visual and spatial working memory. $J$ Exp Psychol Gen, 146(5), 632-650.

Gori, M., Sandini, G., Martinoli, C., \& Burr, D. (2010). Poor haptic orientation discrimination in non-sighted children may reflect disruption of cross-sensory calibration. Curr Biol, 20(3), 223-225.

Holmes, J., \& Adams, J.W. (2006). Working memory and children's mathematical skills: Implications for mathematical development and mathematics curricula. Educational Psychology, 26(3), 339-366.

Holmes, J., Adams, J.W., \& Hamilton, C.J. (2008). The relationship between visuospatial sketchpad capacity and children's mathematical skills. European Journal of Cognitive Psychology, 20(2), 272-289.

Jarvis, H.L., \& Gathercole, S.E. (2003). Verbal and non-verbal working memory and achievements on National Curriculum tests at 11 and 14 years of age. Educational and Child Psychology, 20(3), 123-140.

Kalm, K., \& Norris, D. (2014). The representation of order information in auditory-verbal shortterm memory. J Neurosci, 34(20), 6879-6886.

Kanjlia, S., Feigenson, L., \& Bedny, M. (2018). Numerical cognition is resilient to dramatic changes in early sensory experience. Cognition, 179, 111-120.

Kanjlia, S., Lane, C., Feigenson, L., \& Bedny, M. (2016). Absence of visual experience modifies the neural basis of numerical thinking. Proc Natl Acad Sci U S A, 113(40), 11172-11177.

Lane, C., Kanjlia, S., Omaki, A., \& Bedny, M. (2015). "Visual” cortex of congenitally blind adults responds to syntactic movement. J Neurosci, 35(37), 12859-12868.

Lessard, N., Pare, M., Lepore, F., and Lassonde, M. (1998). Early-blind human subjects localize sound sources better than sighted subjects. Nature, 395, 278-280.

Majerus, S. (2009). Verbal short-term memory and temporary activation of language representations: the importance of distinguishing item and order information. In A. S. Thorn \& M. Page (Eds.), Interactions between short-term and long-term memory in the verbal domain (pp. 244-276). Hove, UK : Psychology Press. 
Majerus, S., Attout, L., Artielle, M.A., \& Van der Kaa, M.A. (2015). The heterogeneity of verbal shirt-term memory impairment in aphasia. Neuropsychologia, 77, 165-176.

Majerus, S., D’Argembeau, A., Perez, T.M, Belayachi, S., Van der Linden, M., et al. (2010). The commonality of neural networks for verbal and visual short-term memory. J Cogn Neurosci, 22(11), 2570-2593.

Majerus, S., Glaser, B., Van der Linden, M., \& Eliez, S. (2006). A multiple case study of verbal short-term memory in velo-cardio-facial syndrome. J Intellect Disabil res, 50(6), 457-469.

Majerus, S., Péters, F., Bouffier, M., Cowan, N., \& Philips, C. (2018). The dorsal attention network reflects both encoding load and top-down control during working memory. $J$ Cogn Neurosci, 30(2), 144-159.

Majerus, S., Van der Linden, M., Braissand, V., \& Eliez, S. (2007). Verbal short-term memory in individuals with chromosome 22q11.2 deletion: specific deficit in serial order retention capacities? Am J Ment Retard, 112(2), 79-93.

Martinez Perez, T., Majerus, S., Mahot, A., \& Poncelet, M. (2012). Evidence for a specific impairment of serial order short-term memory in dyslexic children. Dyslexia, 18(2), 94-109.

Meyer, M.L., Salimpoor, V., Wu, S.S., Geary, D.C., \& Menon, V. (2010). Differential contribution of specific working memory components to mathematics achievement in $2^{\text {nd }}$ and $3^{\text {rd }}$ graders. Learning and individual differences, 20(2), 101-109.

Mix, K.S. (1999). Preschoolers' recognition of numerical equivalence: Sequential sets. J Exp Child Psychol, 74, 309-332.

Mousty, P., Leybaert, J., Alegria, J., Content, A., Morais, J. (1994). BELEC: Batterie d'évaluation du langage écrit. Bruxelles: LAPSE (Université Libre de Bruxelles).

Nieder, A., Diester, I., \& Tudusciuc, O. (2006). Temporal and spatial enumeration processes in the primate parietal cortex. Science, 313, 1431-1435.

Noël, M. P. (2009). Counting on working memory when learning to count and to add: A preschool study. Dev Psychol, 45(6), 1630-1643. 
Noël, M.-P., Seron, X., \& Trovarelli, F. (2004). Working memory as a predictor of addition skills and addition strategies in children. Cahiers de Psychologie Cognitive/Current Psychology of Cognition, $22(1), 3-25$.

Occelli, V., Lacey, S., Stephens, C., Merabet, L.B., \& Sathian, K. (2017). Enhanced verbal abilities in the congenitally blind. Exp Brain Res, 235(6), 1709-1718.

Passolunghi, M..C., Vercelloni, B., \& Schadee, H. (2007). The precursors of mathematics learning: working memory, phonological ability and numerical competence. Cogn Dev, 22(2), 165-184.

Pigeon, C., \& Marin-Lamellet, C. (2015). Evaluation of the attentional capacities and working memory of early and late blind persons. Acta Psychol, 155, 1-7.

Rasmussen, C., \& Bisanz, J. (2005). Representation and working memory in early arithmetic. $J$ Exp Child Psychol, 91(2), 137-157.

Rauschecker, J. P. (1995a). Compensatory plasticity and sensory substitution in the cerebral cortex. Trends Neurosci. 18, 36-43.

Rauschecker, J. P. (1995b). Developmental plasticity and memory. Behav. Brain Res, 66, 7-12.

Raz, N., Striem, E., Pundak, G., Orlov, T., \& Zohary, E. (2007). Superior serial memory in the blind: a case of cognitive compensatory adjustment. Curr Biol, 17(13), 1129-1133.

Reich, L., Szwed, M., Cohen, L., \& Amedi, A. (2011). A ventral visual stream reading center independent of visual experience. Curr Biol, 21(5), 363-368.

Rinaldi, L., Vecchi, T., Fantino, M., Merabet, L. B., \& Cattaneo, Z. (2015). The effect of hand movements on numerical bisection judgments in early blind and sighted individuals. Cortex, 71, 76-84.

Ross, J., \& Burr, D.C. (2010). Vision senses number directly. J Vis, 10(2):10, 1-8.

Salillas, E., Granà, A., El-Yagoubi, R., \& Semenza, C. (2009). Numbers in the blind's « eye ». PlosONE, 4 (7), 1-6.

Seitz, K., \& Schumann-Hengsteler, R. (2000). Mental multiplication and working memory. European Journal of Cognitive Psychology, 12, 552-570.

Simon, T.J. (1999). The foundations of numerical thinking in a brain without numbers. Trends Cogn Sci, 3, 363-365. 
Stoianov, I., \& Zorzi, M. (2012). Emergence of a "visual number sense" in hierarchical generative models. Nat Neurosci, 15(2), 194-196.

Togoli, I., Crollen, V., Arrighi, R., \& Collignon, O. (2020). The shared numerical representation for action and perception develops independently from vision. Cortex, 129, 436-445.

Tokita, M., \& Ishiguchi, A. (2012). Behavioral evidence for format-dependent processes in approximate numerosity representation. Psychon Bull Rev, 19, 285-293.

Voss, P., Lassonde, M., Gougoux, F., Fortin, M., Guillemot, J. P., and Lepore, F. (2004). Earlyand late-onset blind individuals show supra-normal auditory abilities in far-space. Curr Biol, 14, 1734 1738.

Wechsler, D. (2003). The Wechsler intelligence scale for children-fourth edition. London: Pearson. 